\title{
Mechanisms of beneficial effects of metformin on fatty acid-treated human islets
}

\author{
Jing Cen', Ernest Sargsyan1,2, Anders Forslund ${ }^{3}$ and Peter Bergsten1,3 \\ 1Department of Medical Cell Biology, Uppsala University, Uppsala, Sweden \\ ${ }^{2}$ Molecular Neuroscience Group, Institute of Molecular Biology, National Academy of Sciences, Yerevan, Armenia \\ 3Department of Women's and Children's Health, Uppsala University, Uppsala, Sweden \\ Correspondence should be addressed to E Sargsyan: Ernest.Sargsyan@mcb.uu.se
}

\begin{abstract}
Elevated levels of palmitate accentuate glucose-stimulated insulin secretion (GSIS) after short-term and cause beta-cell dysfunction after prolonged exposure. We investigated whether metformin, the first-line oral drug for treatment of T2DM, has beneficial effects on FFA-treated human islets and the potential mechanisms behind the effects. Insulin secretion, oxygen consumption rate (OCR), AMPK activation, endoplasmic reticulum (ER) stress and apoptosis were examined in isolated human islets after exposure to elevated levels of palmitate in the absence or presence of metformin. Palmitate exposure doubled GSIS after 2 days but halved after 7 days compared with control. Inclusion of metformin during palmitate exposure normalized insulin secretion both after 2 and 7 days. After 2-day exposure to palmitate, OCR and the marker of the adaptive arm of ER stress response (sorcin) were significantly raised, whereas AMPK phosphorylation, markers of pro-apoptotic arm of ER stress response ( $p$-EIF2 $\alpha$ and CHOP) and apoptosis (cleaved caspase 3) were not affected. Presence of metformin during 2-day palmitate exposure normalized OCR and sorcin levels. After 7-day exposure to palmitate, OCR and sorcin were not significantly different from control level, p-AMPK was reduced and p-EIF2 $\alpha$, $\mathrm{CHOP}$ and cleaved caspase 3 were strongly upregulated. Presence of metformin during 7-day culture with palmitate normalized the level of p-AMPK, p-EIF2 $\alpha$, CHOP and cleaved caspase 3 but significantly increased the level of sorcin. Our study demonstrates that metformin prevents early insulin hypersecretion and later decrease in insulin secretion from palmitate-treated human islets by utilizing different mechanisms.
\end{abstract}

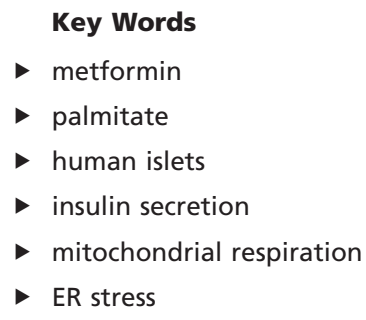

Journal of Molecular Endocrinology (2018) 61, 91-99

\section{Introduction}

Circulating levels of free fatty acids (FFAs) are often elevated in obese subjects (Fraze et al. 1985, Staaf et al. 2016). Such accentuated FFA concentrations contribute to hyperinsulinemia at fasting glucose concentrations and higher insulin levels in response to a glucose challenge (Staaf et al. 2016). In some subjects with long standing obesity, prolonged hyperinsulinemia leads to exhaustion of beta-cells, insufficient secretion of insulin and development of type 2 diabetes mellitus (T2DM) (Felber et al. 1988, Golay \& Ybarra 2005). In line with this, experiments on isolated human islets showed that the fatty acid palmitate treatment accentuates glucosestimulated insulin secretion (GSIS) at earlier time points and causes beta-cell dysfunction after prolonged palmitate exposure (Kristinsson et al. 2013, Staaf et al. 2016, Roomp et al. 2017). The effects of fatty acids on 
insulin secretion have been linked with alterations in insulin biosynthesis and exocytosis (Björklund \& Grill 1999, Kelpe et al. 2003), mitochondrial function (Carlsson et al. 1999), glucose metabolism (Zhou \& Grill 1994), production of reactive oxygen species and ceramides (Carlsson et al. 1999, Manukyan et al. 2015), endoplasmic reticulum (ER) function (Karaskov et al. 2006, Sargsyan et al. 2016) and $G$ protein-coupled receptor signaling (Itoh et al. 2003, Kristinsson et al. 2013). Currently, metformin is recommended as the first-line oral drug for the treatment of T2DM (Chamberlain et al. 2017) with clear benefits in relation to glucose metabolism and diabetes-related complications (Holman et al. 2008). One of the main effects of the drug is to decrease hepatic glucose production, mostly through a mild and transient inhibition of mitochondrial respiratory chain complex I (Viollet et al. 2012). In addition, studies on rat and human islets have provided evidence that metformin has effects on the insulin-producing beta-cells. However, whereas some studies report that metformin restores insulin secretion altered by chronic exposure to high levels of FFAs (Lupi et al. 1999, 2002, Patanè et al. 2000), others show deleterious effects of metformin on beta-cells (Hinke et al. 2007, McKiney et al. 2010, Jiang et al. 2014). Thus, it remains controversial to what extent metformin has direct beneficial effects on beta-cells.

Here, we investigated how metformin influences increased insulin secretion after short-term and decreased insulin secretion after long-term exposure of isolated human islets to elevated levels of palmitate. In addition, we investigated the involvement of various mechanisms such as mitochondrial metabolism, AMPK phosphorylation, ER stress response and apoptosis in metformin-mediated effects.

\section{Materials and methods}

\section{Human islet culture}

Human islets were obtained from the Nordic Network for Clinical Islet Transplantation (Uppsala University Hospital, Uppsala, Sweden). Human islets from braindead non-diabetic donors were used in this study. Human islets were cultured in CMRL 1066 medium (Invitrogen) containing $5.5 \mathrm{mM}$ glucose (Sigma-Aldrich) and supplemented with 10\% fetal bovine serum (Invitrogen), $1 \%$ glutamine (Invitrogen), 100 units $/ \mathrm{mL}$ penicillin (Invitrogen) and $100 \mu \mathrm{g} / \mathrm{mL}$ streptomycin (Invitrogen) at $37^{\circ} \mathrm{C}$ in humidified air containing $5 \% \mathrm{CO}_{2}$. Human islets were treated within 10 days after isolation. Ethical permission to use human islets isolated from human donors has been obtained from the Regional Ethical Review Board in Uppsala (EPN number 2010/006; 201002-10).

\section{Fatty acid and metformin treatment of human islets}

Palmitate (Sigma-Aldrich) was prepared by dissolving in $50 \%$ ethanol to make a stock solution with a concentration of $100 \mathrm{mM}$. The stock solution was then diluted in incubation medium containing $5 \mathrm{mg} / \mathrm{mL}$ fatty acid-free BSA (Boehringer Mannheim GmbH, Germany) to a final concentration of $0.5 \mathrm{mM}$. Palmitate was allowed to complex with $\mathrm{BSA}$ at $37^{\circ} \mathrm{C}$ for at least $30 \mathrm{~min}$. Metformin (Sigma-Aldrich) was prepared in $2 \mathrm{mM}$ stock in distilled water and diluted in incubation medium to a final concentration as stated.

For metformin dose-dependent experiments, human islets were cultured in the absence or presence of 10, 15, 25 or $100 \mu \mathrm{M}$ metformin for 7 days. GSIS, insulin content and AMPK phosphorylation were measured after treatment.

Human islets were exposed to $0.5 \mathrm{mM}$ palmitate in the absence or presence of $25 \mu \mathrm{M}$ metformin for 0,2 days or 7 days. Human islets were also cultured in the absence or presence of $25 \mu \mathrm{M}$ metformin alone for 0,2 days or 7 days. GSIS, insulin content, oxygen consumption rate (OCR), AMPK phosphorylation, ER stress (p-eIF2a and $\mathrm{CHOP})$, sorcin and cleaved caspase- 3 protein expression were measured after treatment.

Between four and nine biological replicates (donors) were used for each set of experiments.

\section{GSIS and insulin content from human islets}

Human islets were perifused as described previously (Bergsten 1995). Briefly, around 15 human islets were hand-picked and placed into a perifusion chamber. Islets were perifused for $60 \mathrm{~min}$ at $37^{\circ} \mathrm{C}$ in $\mathrm{KRBH}$ buffer supplemented with $1 \mathrm{mg} / \mathrm{mL}$ BSA and $2 \mathrm{mM}$ glucose. After this initial perifusion period, samples were collected every $5 \mathrm{~min}$ for $20 \mathrm{~min}$ at the same concentration of glucose. This was followed by another 20-min perifusion with the same buffer containing $1 \mathrm{mg} / \mathrm{mL}$ BSA and $20 \mathrm{mM}$ glucose. Perifusates were collected at 2, 4, 6, 10, 15 and $20 \mathrm{~min}$. The perifusion rate was $170 \mu \mathrm{L} / \mathrm{min}$ and collected perifusates were used to measure the amounts of secreted insulin. After perifusion, islets were washed with Dulbecco's Phosphate-Buffered Saline (DPBS) and lysed in DPBS containing 1\% Triton X-100 (Sigma-Aldrich). Lysates were used for measurements of insulin content and protein content. 
The amount of secreted insulin and insulin content were measured by enzyme-linked immunosorbent assay as described previously (Bergsten \& Hellman 1993). Total protein content in the lysates was determined by DC protein assay according to manufacturer's instructions (Bio-Rad). For each perifusion, total insulin secretion during $20 \mathrm{mM}$ glucose was normalized to total protein content. Insulin content was also normalized to total protein content. Insulin secretion and insulin content were expressed as fold change between treated and untreated islets for each donor.

\section{Measurement of mitochondrial function}

OCR of isolated human islets was determined by Extracellular Flux Analyzer XFe96 (Seahorse Bioscience, MA, USA) as previously reported (Cen et al. 2016). Ten hand-picked human islets were placed into the wells of the XFe 96 cell culture microplate pre-coated with $3 \%$ ECM gel (Sigma-Aldrich) and $5 \mu \mathrm{g} / \mathrm{mL}$ fibronectin (Sigma-Aldrich). Islets were pre-incubated with assay medium containing $5.5 \mathrm{mM}$ glucose $(\mathrm{pH}$ adjusted to 7.4) for $1 \mathrm{~h}$ at $37^{\circ} \mathrm{C}$ in the air before the microplate was inserted into the Analyzer. For each donor, 6-8 replicates of each treatment condition were used. OCR was then measured for $40 \mathrm{~min}$. The proportions of respiration driving ATP synthesis and proton leak were determined by blocking ATP synthase by injection of $5 \mu \mathrm{M}$ oligomycin (Sigma-Aldrich). Subsequently, $5 \mu \mathrm{M}$ of the uncoupler cyanide-p-trifluoromethoxyphenylhydrazone (FCCP) (Sigma-Aldrich) was added to determine the maximal respiratory capacity. Lastly, $5 \mu \mathrm{M}$ rotenone (Sigma-Aldrich) and $5 \mu \mathrm{M}$ antimycin $\mathrm{A}$ (Sigma-Aldrich) were added together to block transfer of electrons from complex I and complex III of the respiratory chain to measure the remaining nonmitochondrial-dependent respiration. To calculate the mitochondrial respiration, non-mitochondrial OCR was subtracted from the total OCR. Extracellular acidification rate (ECAR) was measured in parallel and calculated under $5.5 \mathrm{mM}$ glucose treatment. Data were normalized to total islet area calculated by the ImageJ software (National Institutes of Health) from pictures (40x) taken with camera (Olympus) mounted onto an inverted Olympus CKX41 microscope (Olympus).

\section{Protein expression}

Expression of specific proteins in human islets was determined by western blot analysis. Samples were prepared by washing the human islets with DPBS for 5 times followed by sonicating in DPBS containing 1\% Triton X-100 and protease inhibitory cocktail (Sigma Aldrich) on ice. After lysing, samples were centrifuged and the protein concentration was measured by DC protein assay (Bio-Rad). Then, the samples were electrophoresed and transferred onto PVDF-membrane (Bio-Rad) as described previously (Sargsyan et al. 2016). Immunoblotting was performed with primary antibodies against phospho-AMPK $\alpha$ (Cell Signaling Technology Cat\# 2531, RRID:AB_330330), AMPK $\alpha$ (Cell Signaling Technology Cat\# 2603, RRID:AB_490795), phospho-eIF2 $\alpha$ (Cell Signaling Technology Cat\# 3597, RRID:AB_390740), CHOP/GADD153 (Santa Cruz Biotechnology Cat\# sc-575, RRID:AB_631365), cleaved caspase-3 (Cell Signaling Technology Cat\# 9661, RRID:AB_2341188), sorcin (Thermo Fisher Scientific Cat\# PA5-23143, RRID:AB_2540670) and $\beta$-actin (Cell Signaling Technology Cat\# 4970, RRID:AB_2223172) and secondary antibody, mouse antirabbit IgG-HRP (Santa Cruz Biotechnology Cat\# sc-2357, RRID:AB_628497). Immunoreactive bands were detected by enhanced chemiluminescence (ECL) detection kit (Amersham Biosciences GE) and imaged with ChemiDoc XRS+ (Bio-Rad). Signals were quantified with Image Lab 4.0.1 (Bio-Rad). Quantitative analysis of bands densities was normalized to $\beta$-actin unless otherwise stated. Data were expressed as fold change between treated and untreated islets for each donor.

\section{Statistical analysis}

Results were presented as means \pm s.E.M. Statistical analysis was performed using GraphPad Prism Version 6.0c (GraphPad software). Statistical significance among several groups was analyzed by using one-way ANOVA followed by Holm-Sidak multiple comparison test. $P<0.05$ was considered statistically significant.

\section{Results}

\section{Effect of metformin on GSIS and insulin content from isolated human islets exposed to palmitate}

GSIS and intracellular insulin content were measured from isolated human islets cultured in the presence of palmitate for 0,2 and 7 days. Palmitate caused timedependent changes in GSIS and insulin content as previously demonstrated (Staaf et al. 2016). Compared with control islets, GSIS was almost doubled after culturing in the presence of palmitate for 2 days (Fig. 1A and B). After 7-day exposure to palmitate, GSIS was reduced to 
approximately $50 \%$ of the control level. Insulin content was reduced by $25 \%$ after 2 -day and $75 \%$ after 7 -day exposure to palmitate, respectively (Fig. 1C).

Next, we investigated whether metformin influenced insulin secretion and insulin content from human islets exposed to or not to elevated levels of palmitate for 2 and 7 days. In the study, we used $25 \mu \mathrm{M}$ metformin, a concentration that has been optimized in preliminary experiments (Supplementary Fig. 1, see section on supplementary data given at the end of this article). Inclusion of $25 \mu \mathrm{M}$ metformin during 2-day palmitate exposure reduced insulin secretion to control level (Fig. 1A and B). When metformin was included during 7-day palmitate culture, insulin secretion was significantly increased reaching the control level (Fig. 1A and B). When insulin content was measured in the presence of metformin, we observed no changes after 2-day treatment but more than 2-fold increase after 7-day treatment compared to corresponding treatment with palmitate alone (Fig. 1C). In the absence of palmitate, metformin did not affect insulin secretion after 2 days but reduced the level by around 20\% compared with control islets after 7 days (Supplementary Fig. 2A and B).

\section{Effect of metformin on mitochondrial metabolism of palmitate-treated human islets}

We have previously demonstrated that palmitate can enhance mitochondrial function in human islets and beta-cells (Kristinsson et al. 2015, Cen et al. 2016). On the other hand, it is known that metformin can inhibit mitochondrial respiration chain complex I (Viollet et al. 2012). Therefore, we examined how the drug affected mitochondrial function in fatty acidtreated islets. Palmitate alone caused a $65 \%$ rise in OCR after 2 days (Fig. $2 \mathrm{~A}$ and $\mathrm{B}$ ). The rise was mainly due to changes in ATP-coupled OCR and not proton leak OCR (Fig. 2C and D). Also, maximal OCR, gained from the introduction of FCCP, was increased by $20 \%$ (Fig. 2E). When metformin was also included during culture with palmitate, mitochondrial OCR was decreased to control level (Fig. 2A and B). The effect was due to inhibition of ATP-coupled OCR. Maximal OCR was also normalized (Fig. 2E). After 7-day exposure to palmitate, there was no significant difference in mitochondrial OCR compared with control islets (Fig. 2A and B). Maximal OCR was attenuated to $70 \%$ of control level, however (Fig. 2E). Inclusion of metformin during 7-day palmitate culture showed no effect on mitochondrial OCR but restored maximal respiration rate (Fig. 2E). ECAR, an indicator of
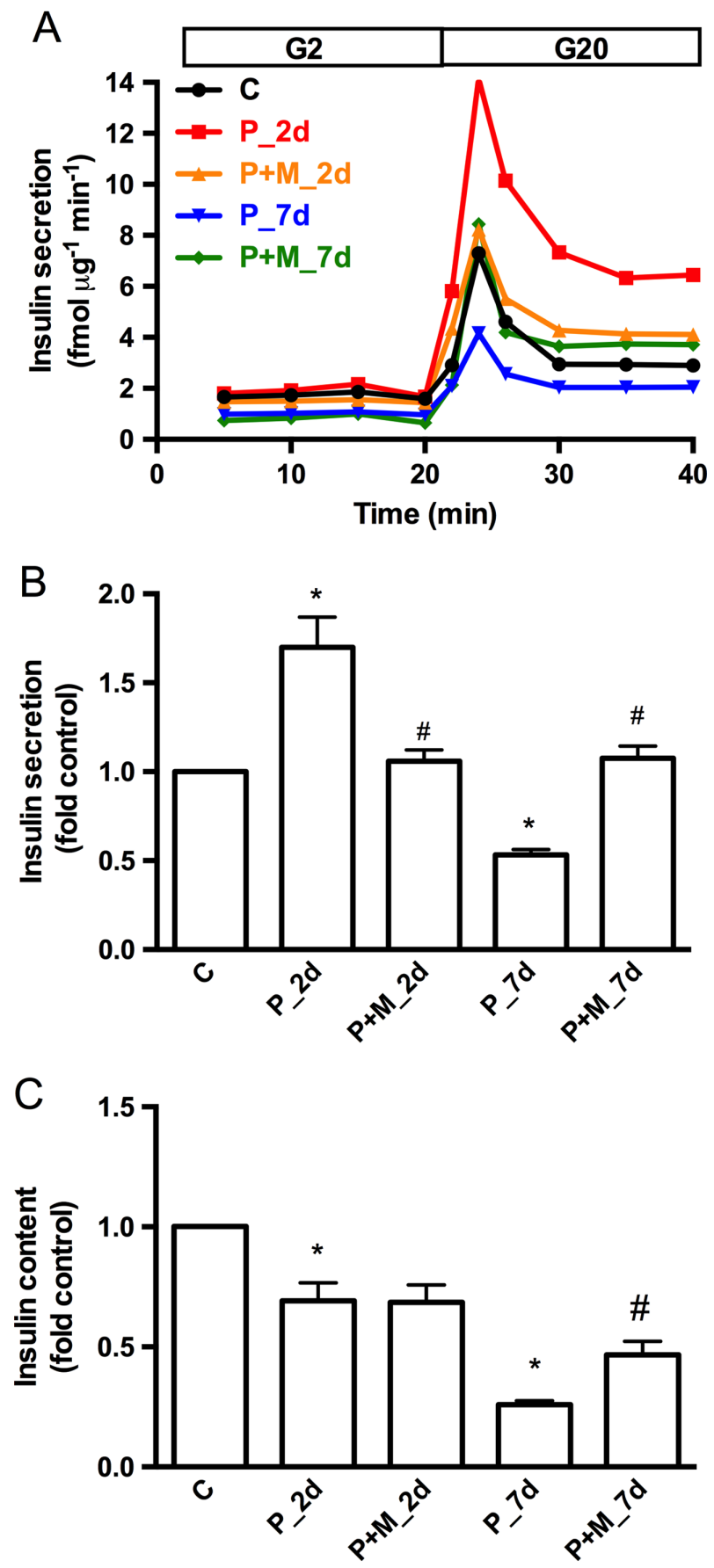

Figure 1

Effect of metformin on GSIS and insulin content from human islets exposed to palmitate. Human islets were cultured in the absence (C) or presence of palmitate $(P)$ with or without metformin (M) for 2 days (P_2d, $\left.P+M \_2 d\right)$ and 7 days $\left(P_{-} 7 d, P+M \_7 d\right)$. After culture, human islets were perifused for $1 \mathrm{~h}$ with $2 \mathrm{mM}$ glucose (G2) followed by $20 \mathrm{~min}$ of perifusion with $20 \mathrm{mM}$ glucose (G20). Representative graphs are shown (Panel A). Total insulin secretion at $20 \mathrm{mM}$ glucose during $20 \mathrm{~min}$ was calculated, normalized to protein and expressed as fold control (Panel B). After perifusion, islets were lysed and insulin content was normalized to total protein content and expressed as fold control (Panel C). Results are means \pm S.E.M. of $6-7$ donors. ${ }^{*} P<0.05$ vs $C ;{ }^{*} P<0.05$ vs $P$ at the corresponding time point. 

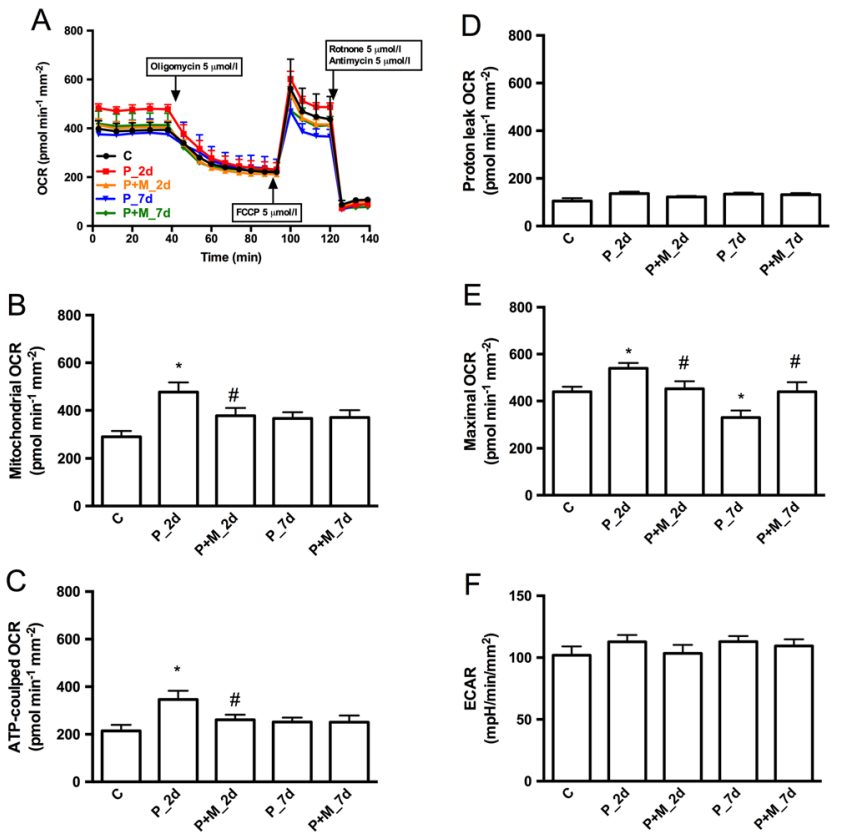

Figure 2

Effect of metformin on mitochondrial metabolism in human islets exposed to palmitate. Human islets were cultured in the absence (C) or presence of palmitate $(P)$ with or without metformin $(M)$ for 2 days $\left(P \_2 d\right.$, $\left.\mathrm{P}+\mathrm{M} \_2 \mathrm{~d}\right)$ and 7 days ( $\mathrm{P}$-7d, $\mathrm{P}+\mathrm{M}$ _7d). After culture, oxygen consumption rate (OCR) and extracellular acidification rate (ECAR) were measured. OCR from a representative experiment, where each point is a mean \pm S.E.M. of 6-8 replicates from one donor, is shown (Panel A). The injection of $5 \mu \mathrm{M}$ oligomycin, $5 \mu \mathrm{M} \mathrm{FCCP}$ and combination of $5 \mu \mathrm{M}$ of rotenone and $5 \mu \mathrm{M}$ antimycin are shown as indicated (Panel A). Mitochondrial OCR was calculated by subtracting non-mitochondrial OCR from the total OCR (Panel B). ATP-coupled (Panel C) and proton leak (Panel D) portions of mitochondrial OCR were calculated based on the effect of oligomycin. Maximal OCR (Panel E) was determined by analyzing the effect of FCCP. ECAR measurements (Panel F) were taken in parallel. Results are means \pm S.E.M. of $4-6$ donors. ${ }^{*} P<0.05$ vs $C ;{ }^{\#} P<0.05$ vs $P$ at the corresponding time point.

glycolysis, was not affected by palmitate irrespective of the absence or presence of metformin (Fig. 2F). Metformin alone showed no effect on OCR after 2 days but slightly decreased it after 7 days (Supplementary Fig. 2D, E and F). ECAR was slightly decreased both after 2 and 7 days of metformin treatment alone (Supplementary Fig. 2I).

\section{Effect of metformin on phosphorylation of AMPK in palmitate-treated human islets}

Long-term palmitate exposure has been linked to decreased phosphorylation of AMPK in beta-cells (Sun et al. 2008). Metformin increases AMP/ATP ratio, which leads to increased phosphorylation and activation of AMPK (El-Mir et al. 2000). Thus, we investigated how palmitate alone and in combination with metformin affects the phosphorylation of AMPK in human islets.
Human islets cultured with palmitate for 2 days showed no effect on p-AMPK/AMPK (Fig. 3). Surprisingly, addition of metformin had no effect on p-AMPK/AMPK ratio after 2-day palmitate treatment. When human islets were cultured with palmitate for 7 days, p-AMPK/AMPK was reduced by $\sim 50 \%$ compared with the control islets (Fig. 3). The addition of metformin in the presence of palmitate restored this ratio to the control level after 7 days. When islets were cultured in the presence of metformin alone, rise in AMPK phosphorylation was observed both after 2- and 7-day exposure to metformin (Supplementary Fig. 2J).

\section{Effect of metformin on ER stress and apoptosis in palmitate-treated human islets}

Long-term exposure to saturated fatty acids causes ER stress and apoptosis in beta-cells (Sargsyan et al. 2008). Metformin has been shown to protect beta-cells and human pancreatic islets against lipotoxicity (Lupi et al. 2002, Marchetti et al. 2004). We explored whether metformin attenuates the ER stress response and apoptosis in palmitate-treated human islets. ER stress markers, p-eIF2 $\alpha$, CHOP, calcium-binding protein, sorcin and apoptosis marker, cleaved caspase-3, were measured by western blot after 2- and 7-day treatments. After 2 days,
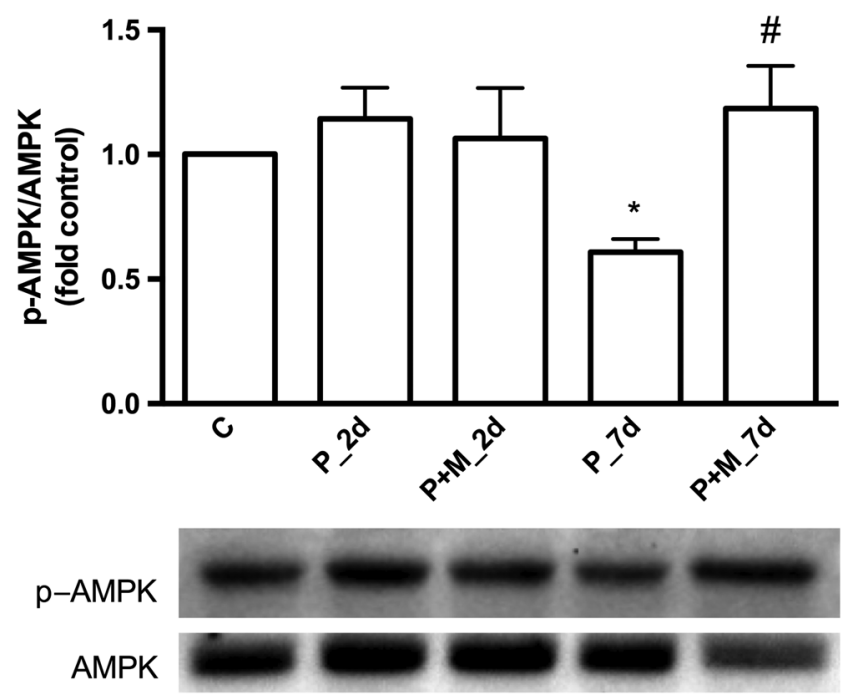

Figure 3

Effect of metformin on phosphorylation of AMPK in human islets exposed to palmitate. Human islets were cultured in the absence (C) or presence of palmitate $(P)$ with or without metformin (M) for 2 days (P_2d, $\left.P+M \_2 d\right)$ and 7 days (P_7d, $\left.P+M \_7 d\right)$. After culture, human islets were lysed, and the levels of $\mathrm{p}$-AMPK and AMPK were measured by western blotting. p-AMPK/AMPK ratio was calculated and expressed as fold control. Results are mean \pm s.E.M. of nine donors. ${ }^{*} P<0.05$ vs $C$; $\# P<0.05$ vs P_7d. 
palmitate caused no significant changes in the expression of ER stress markers p-eIF $2 \alpha$ and CHOP but increased the $\mathrm{Ca}^{2+-b i n d i n g}$ protein sorcin to 3 -fold compared with the control human islets (Fig. 4A, B, and C). Cleaved caspase-3 levels were not affected by palmitate in islets exposed to palmitate for 2 days (Fig. 4D). When metformin was included, p-eIF $2 \alpha$ and CHOP were not affected, but sorcin levels were reduced to 1.7-fold in islets exposed to palmitate for 2 days (Fig. 4A, B, and C). Cleaved caspase-3 levels were not affected by metformin (Fig. 4D). After 7 days, protein levels of ER stress markers p-eIF2 $\alpha$, CHOP and sorcin were $\sim 1.7$-fold in islets cultured in the presence of palmitate compared to control islets. Cleaved caspase- 3 levels were significantly increased by palmitate (Fig. 4D). When metformin was included during culture expression, levels of p-eIF2 $\alpha$ and CHOP were reduced to levels observed in control islets but sorcin levels were increased to three-fold. The elevated cleaved caspase- 3 levels were normalized by metformin in islets cultured for 7 days in the presence of palmitate (Fig. 4D). Metformin alone did not cause any significant changes in the expression levels of p-EIF $2 \alpha$, sorcin and active caspase- 3 but slightly decreased expression of CHOP after 7-day treatment (Supplementary Fig. 2K, L, M and N).

\section{Discussion}

Metformin is one of the major drugs, which is used for treatment of patients with T2DM, in particular overweight and obese individuals (Inzucchi et al. 2015, Chamberlain et al. 2017). The drug has beneficial effect on improving not only blood glucose concentration (Holman et al. 2008), but also lipid profiles (Pentikäinen et al. 1990). However, knowledge about the precise molecular mechanism of action of metformin is still limited. Moreover, the direct effect of metformin on beta-cells is still controversial. In the present study, we investigated the effect of metformin on isolated human islets exposed to high levels of palmitate for short and long time periods.

We used $25 \mu \mathrm{M}$ metformin since at this concentration the drug mildly decreased GSIS but did not affect intracellular insulin content after 7-day culture suggesting that the concentration is effective but not toxic. In addition, this concentration is around the reported plasma concentrations of metformin $(8-24 \mu \mathrm{M})$ in humans treated with metformin (Tucker et al. 1981, Gormsen et al. 2016). We discovered that metformin normalized both the increased insulin secretion observed after 2-day palmitate treatment and the decreased insulin secretion observed after 7-day palmitate treatment. These findings raised the
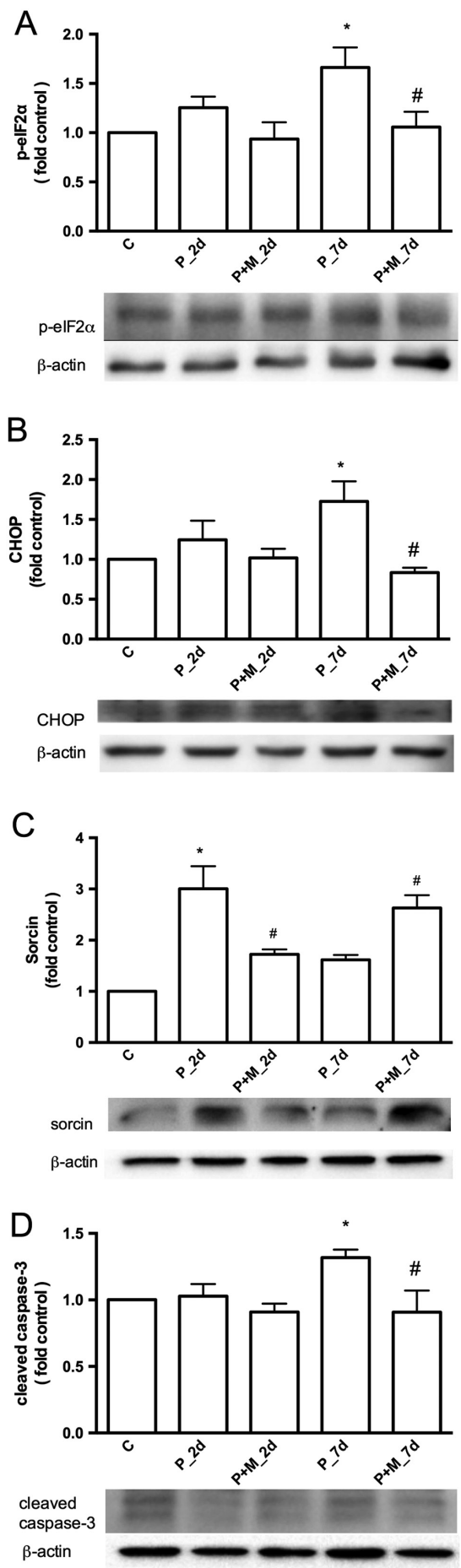

Figure 4

Effect of metformin on endoplasmic reticulum (ER) stress and apoptotic markers in human islets exposed to palmitate. Human islets were cultured in the absence $(C)$ or presence of palmitate $(P)$ with or without metformin (M) for 2 days (P_2d, P+M_2d) and 7 days (P_7d, P+M_7d). After culture, expression of ER stress markers, p-elF $2 \alpha$ (Panel A), CHOP (Panel B), calcium-binding protein, sorcin (Panel C), and apoptotic marker, cleaved caspase-3 (Panel D), were determined by Western blot. Protein levels were normalized to $\beta$-actin and expression as fold control. Results are means \pm S.E.M. of $4-6$ donors. ${ }^{*} P<0.05$ vs $C ;{ }^{\#} P<0.05$ vs $P \_7 d$. 
question about the potential mechanisms behind these effects. We hypothesized that changes in mitochondrial metabolism, AMPK phosphorylation, ER stress response and apoptosis, which are known to be affected by fatty acids and metformin, were implicated in the observed beneficial effects of the drug.

It is known that metformin exerts a mild and reversible effect on mitochondrial respiratory chain complex I, which results in reduced ATP production (Owen et al. 2000, Zhou et al. 2001). At the same time, we and others have previously reported that palmitate alters mitochondrial metabolism both after short- and long-term exposure of beta-cells or human islets to fatty acids (Patanè et al. 2000, Kristinsson et al. 2015, 2017, Cen et al. 2016). Therefore, as a first step, we examined how metformin affects mitochondrial metabolism in palmitate-treated human islets. In line with our previous findings (Kristinsson et al. 2015, Cen et al. 2016), palmitate increased mitochondrial respiration after 2-day culture. Considering the importance of ATP generation in the regulation of insulin secretion, the detected effects of palmitate on mitochondrial metabolism may explain increased GSIS after 2-day culture with palmitate. Metformin affected human islets exposed to palmitate by inhibiting ATP-coupled OCR (but not proton leak OCR) after 2-day palmitate exposure suggesting that reduced generation of ATP is a potential mechanism of how metformin lowers GSIS. Importantly, the metformin concentration chosen did not affect OCR after culturing the islets for 2 days in the absence of palmitate. After 7-day palmitate treatment, ATP generation was not different from control human islets. Instead, the fatty acid altered the maximal respiration capacity. Presence of metformin during 7-day palmitate exposure did not affect ATP generation but improved the maximal respiration capacity. Nevertheless, absence of changes in ATP generation after 7 days suggests that mitochondria do not play a direct role in the observed effects of palmitate and metformin on insulin secretion after 7 days. As expected, in islets cultured for 7 days in the presence of metformin alone, OCR was mildly reduced (Owen et al. 2000, Zhou et al. 2001).

Changes in ATP generation is anticipated to affect the activity of AMP-activated protein kinase (AMPK), a protein that plays an important role in sensing cellular energy and controlling metabolic homeostasis (Owen et al. 2000, Zhou et al. 2001). It has been reported that acute exposure of beta-cells to palmitate induces AMPK activation and augments insulin secretion, whereas chronic palmitate exposure attenuates phosphorylation of AMPK and decreases GSIS (Wang et al. 2007, Sun et al. 2008). However, the role of AMPK in beta-cells under chronic lipotoxicity is poorly understood. In our study, islets exposed to palmitate and metformin for 2 days showed no effects on phosphorylation of AMPK. After 7 days, AMPK phosphorylation was significantly diminished in islets exposed to palmitate alone but restored when metformin was also present. The fact that changes in insulin secretion from islet exposed to palmitate and metformin follow the changes in AMPK phosphorylation after 7 but not 2 days suggests that this mechanism plays an important role in regulation of GSIS from human islets after prolonged exposure to the fatty acid. Why changes in ATP generation observed after 2 days are conversed into changes in phosphorylation of AMPK only after 7 days remains to be investigated. In the absence of palmitate, the drug caused significant rise in AMPK phosphorylation, which is in agreement with previous studies on beta-cell lines and human skeletal muscles (Musi et al. 2002, Jiang et al. 2014).

Another mechanism of how fatty acids affects betacells function is the increase in ER stress and apoptosis in beta-cells (Sargsyan et al. 2008, Kristinsson et al. 2013, Staaf et al. 2016). Metformin has been shown to attenuate palmitate-induced ER stress and apoptosis in rat betacells (Simon-Szabó et al. 2014). After 2-day exposure of human islets to palmitate, there were no changes in the expression level of pro-apoptotic markers of ER stress response, phosphorylated eIF2a and CHOP; and inclusion of metformin did not affect their expression levels. After 7-day palmitate treatment, the markers of ER stress and apoptosis were upregulated in the absence and normalized in the presence of metformin, which was in line with the previous study (Simon-Szabó et al. 2014). We have previously demonstrated that one of the major mechanisms behind palmitate toxicity is inadequate activation of adaptive pathways of ER stress response (Sargsyan et al. 2016). Stimulation of adaptive mechanisms protected beta-cells from palmitate-induced ER stress and apoptosis (Sargsyan et al. 2016). Therefore, here we also measured the levels of sorcin, a $\mathrm{Ca}^{2+-b i n d i n g}$ protein that relocates calcium from the cytoplasm to the ER and, in such way, protects cells from ER stress (Maki et al. 2002, Marmugi et al. 2016). The substantial increase of sorcin after 2-day palmitate culture of human islets reflects an adaptation against palmitate-induced ER stress (Cunha et al. 2008). But this compensatory effect failed in a long-term period resulting in upregulation of ER stress makers. The introduction of metformin during palmitate culture delayed the development of ER stress and, as a 
result, the adaptive effects were also postponed. Our data indicate that the reduction in ER stress and apoptosis by metformin treatment may contribute to restoration of insulin secretion and intracellular insulin content from human islets after 7-day culture with palmitate.

In conclusion, our study demonstrates that metformin prevents early insulin hypersecretion and later decrease in insulin secretion from palmitate-treated human islets by utilizing different mechanisms.

\section{Supplementary data}

This is linked to the online version of the paper at https://doi.org/10.1530/ JME-17-0304.

\section{Declaration of interest}

The authors declare that there is no conflict of interest that could be perceived as prejudicing the impartiality of the research reported.

\section{Funding}

This work was supported by the European Commission FP7 Project BetaJUDO (grant number 279 153), Swedish Diabetes Association (grant number DIA 2016-146) and Family Ernfors Foundation (grant number 170504).

\section{Author contribution statement}

J C designed and performed experiments, analyzed the data, prepared the figures and wrote the manuscript. E R contributed to the design of the study, to the analysis of the data and to the discussion and writing of the manuscript. A F and P B contributed to the design of the study and to the discussion and writing of the manuscript. All authors corrected, revised and approved the final version of the manuscript.

\section{Acknowledgements}

Human islets were generously provided by the Nordic Network for Clinical Islet Transplantation.

\section{References}

Bergsten P 1995 Slow and fast oscillations of cytoplasmic Ca2+ in pancreatic islets correspond to pulsatile insulin release. American Journal of Physiology 268 282-287. (https://doi.org/10.1152/ ajpendo.1995.268.2.E282)

Bergsten P \& Hellman B 1993 Glucose-induced amplitude regulation of pulsatile insulin secretion from individual pancreatic islets. Diabetes 42 670-674. (https://doi.org/10.2337/diab.42.5.670)

Björklund A \& Grill V 1999 Enhancing effects of long-term elevated glucose and palmitate on stored and secreted proinsulin-to-insulin ratios in human pancreatic islets. Diabetes 48 1409-1414.

Carlsson C, Borg LA \& Welsh N 1999 Sodium palmitate induces partial mitochondrial uncoupling and reactive oxygen species in rat pancreatic islets in vitro. Endocrinology 140 3422-3428. (https://doi. org/10.1210/endo.140.8.6908)

Cen J, Sargsyan E \& Bergsten P 2016 Fatty acids stimulate insulin secretion from human pancreatic islets at fasting glucose concentrations via mitochondria-dependent and -independent mechanisms. Nutrition and Metabolism 13 59-67.

Chamberlain JJ, Herman WH, Leal S, Rhinehart AS, Shubrook JH, Skolnik N \& Kalyani RR 2017 Pharmacologic therapy for type 2 diabetes: synopsis of the 2017 American Diabetes Association Standards of Medical Care in Diabetes. Annals of Internal Medicine 166 572-578. (https://doi.org/10.7326/M16-2937)

Cunha DA, Hekerman P, Ladrière L, Bazarra-Castro A, Ortis F, Wakeham MC, Moore F, Rasschaert J, Cardozo AK, Bellomo E, et al. 2008 Initiation and execution of lipotoxic ER stress in pancreatic beta-cells. Journal of Cell Science 121 2308-2318. (https://doi. org/10.1242/jcs.026062)

El-Mir MY, Nogueira V, Fontaine E, Avéret N, Rigoulet M \& Leverve X 2000 Dimethylbiguanide inhibits cell respiration via an indirect effect targeted on the respiratory chain complex I. Journal of Biological Chemistry 275 223-228. (https://doi.org/10.1074/ jbc.275.1.223)

Felber JP, Golay A, Jéquier E, Curchod B, Temler E, DeFronzo RA \& Ferrannini E 1988 The metabolic consequences of long-term human obesity. International Journal of Obesity 12 377-389.

Fraze E, Donner CC, Swislocki AL, Chiou YA, Chen YD \& Reaven GM 1985 Ambient plasma free fatty acid concentrations in noninsulindependent diabetes mellitus: evidence for insulin resistance. Journal of Clinical Endocrinology and Metabolism 61 807-811. (https://doi. org/10.1210/jcem-61-5-807)

Golay A \& Ybarra J 2005 Link between obesity and type 2 diabetes. Best Practice and Research: Clinical Endocrinology and Metabolism 19 649-663.

Gormsen LC, Sundelin EI, Jensen JB, Vendelbo MH, Jakobsen S, Munk OL, Hougaard Christensen MM, Brøsen K, Frøkiær J \& Jessen N 2016 In vivo imaging of human 11C-metformin in peripheral organs: dosimetry, biodistribution, and kinetic analyses. Journal of Nuclear Medicine: Official Publication, Society of Nuclear Medicine 57 1920-1926. (https://doi.org/10.2967/ jnumed.116.177774)

Hinke SA, Martens GA, Cai Y, Finsi J, Heimberg H, Pipeleers D \& Van de Casteele M 2007 Methyl succinate antagonises biguanide-induced AMPK-activation and death of pancreatic beta-cells through restoration of mitochondrial electron transfer. British Journal of Pharmacology 150 1031-1043. (https://doi.org/10.1038/sj. bjp.0707189)

Holman RR, Paul SK, Bethel MA, Matthews DR \& Neil HAW 2008 10year follow-up of intensive glucose control in type 2 diabetes. New England Journal of Medicine 359 1577-1589. (https://doi.org/10.1056/ NEJMoa0806470)

Inzucchi SE, Bergenstal RM, Buse JB, Diamant M, Ferrannini E, Nauck M, Peters AL, Tsapas A, Wender R \& Matthews DR 2015 Management of hyperglycemia in type 2 diabetes, 2015: a patient-centered approach: update to a position statement of the American Diabetes Association and the European Association for the Study of Diabetes. Diabetes Care 38 140-149. (https://doi.org/10.2337/dc14-2441)

Itoh Y, Kawamata Y, Harada M, Kobayashi M, Fujii R, Fukusumi S, Ogi K, Hosoya M, Tanaka Y, Uejima H, et al. 2003 Free fatty acids regulate insulin secretion from pancreatic beta cells through GPR40. Nature 422 173-176. (https://doi.org/10.1038/nature01478)

Jiang Y, Huang W, Wang J, Xu Z, He J, Lin X, Zhou Z \& Zhang J 2014 Metformin plays a dual role in MIN6 pancreatic $\beta$ cell function through AMPK-dependent autophagy. International Journal of Biological Sciences 10 268-277. (https://doi.org/10.7150/ijbs.7929)

Karaskov E, Scott C, Zhang L, Teodoro T, Ravazzola M \& Volchuk A 2006 Chronic palmitate but not oleate exposure induces endoplasmic reticulum stress, which may contribute to INS-1 pancreatic beta-cell apoptosis. Endocrinology 147 3398-3407. (https:// doi.org/10.1210/en.2005-1494)

Kelpe CL, Moore PC, Parazzoli SD, Wicksteed B, Rhodes CJ \& Poitout V 2003 Palmitate inhibition of insulin gene expression is mediated at 
the transcriptional level via ceramide synthesis. Journal of Biological Chemistry 278 30015-30021. (https://doi.org/10.1074/jbc. M302548200)

Kristinsson H, Smith DM, Bergsten P \& Sargsyan E 2013 FFAR1 is involved in both the acute and chronic effects of palmitate on insulin secretion. Endocrinology 154 4078-4088. (https://doi. org/10.1210/en.2013-1352)

Kristinsson H, Bergsten P \& Sargsyan E 2015 Free fatty acid receptor 1 (FFAR1/GPR40) signaling affects insulin secretion by enhancing mitochondrial respiration during palmitate exposure. Biochimica Et Biophysica Acta 1853 3248-3257. (https://doi.org/10.1016/j. bbamcr.2015.09.022)

Kristinsson H, Sargsyan E, Manell H, Smith DM, Göpel SO \& Bergsten P 2017 Basal hypersecretion of glucagon and insulin from palmitateexposed human islets depends on FFAR1 but not decreased somatostatin secretion. Scientific Reports 7 4657. (https://doi. org/10.1038/s41598-017-04730-5)

Lupi R, Del Guerra S, Tellini C, Giannarelli R, Coppelli A, Lorenzetti M, Carmellini M, Mosca F, Navalesi R \& Marchetti P 1999 The biguanide compound metformin prevents desensitization of human pancreatic islets induced by high glucose. European Journal of Pharmacology 364 205-209. (https://doi.org/10.1016/S00142999(98)00807-3)

Lupi R, Del Guerra S, Fierabracci V, Marselli L, Novelli M, Patanè G, Boggi U, Mosca F, Piro S, Del Prato S, et al. 2002 Lipotoxicity in human pancreatic islets and the protective effect of metformin. Diabetes 51 (Supplement 1) S134-S137. (https://doi.org/10.2337/ diabetes.51.2007.S134)

Maki M, Kitaura Y, Satoh H, Ohkouchi S \& Shibata H 2002 Structures, functions and molecular evolution of the penta-EF-hand Ca2+binding proteins. Biochimica Et Biophysica Acta 1600 51-60. (https:// doi.org/10.1016/S1570-9639(02)00444-2)

Manukyan L, Ubhayasekera SJKA, Bergquist J, Sargsyan E \& Bergsten P 2015 Palmitate-induced impairments of $\beta$-cell function are linked with generation of specific ceramide species via acylation of sphingosine. Endocrinology 156 802-812. (https://doi.org/10.1210/ en.2014-1467)

Marchetti P, Del Guerra S, Marselli L, Lupi R, Masini M, Pollera M, Bugliani M, Boggi U, Vistoli F, Mosca F, et al. 2004 Pancreatic islets from type 2 diabetic patients have functional defects and increased apoptosis that are ameliorated by metformin. Journal of Clinical Endocrinology and Metabolism 89 5535-5541. (https://doi. org/10.1210/jc.2004-0150)

Marmugi A, Parnis J, Chen X, Carmichael L, Hardy J, Mannan N, Marchetti P, Piemonti L, Bosco D, Johnson P, et al. 2016 Sorcin links pancreatic $\beta$-Cell lipotoxicity to ER Ca2+ stores. Diabetes 65 1009-1021. (https://doi.org/10.2337/db15-1334)

McKiney JM, Irwin N, Flatt PR, Bailey CJ \& McClenaghan NH 2010 Acute and long-term effects of metformin on the function and insulin secretory responsiveness of clonal $\beta$-cells. Biological Chemistry 391 1451-1459. (https://doi.org/10.1515/bc.2010.139)

Musi N, Hirshman MF, Nygren J, Svanfeldt M, Bavenholm P, Rooyackers O, Zhou G, Williamson JM, Ljunqvist O, Efendic S, et al. 2002 Metformin increases AMP-activated protein kinase activity in skeletal muscle of subjects with type 2 diabetes. Diabetes 51 2074-2081. (https://doi.org/10.2337/ diabetes.51.7.2074)

Owen MR, Doran E \& Halestrap AP 2000 Evidence that metformin exerts its anti-diabetic effects through inhibition of complex 1 of the mitochondrial respiratory chain. Biochemical Journal 348 607-614. (https://doi.org/10.1042/bj3480607)

Patanè G, Piro S, Rabuazzo AM, Anello M, Vigneri R \& Purrello F 2000 Metformin restores insulin secretion altered by chronic exposure to free fatty acids or high glucose: a direct metformin effect on pancreatic beta-cells. Diabetes 49 735-740.

Pentikäinen PJ, Voutilainen E, Aro A, Uusitupa M, Penttilä I \& Vapaatalo H 1990 Cholesterol lowering effect of metformin in combined hyperlipidemia: placebo controlled double blind trial. Annals of Medicine 22 307-312.

Roomp K, Kristinsson H, Schvartz D, Ubhayasekera K, Sargsyan E, Manukyan L, Chowdhury A, Manell H, Satagopam V, Groebe K, et al. 2017 Combined lipidomic and proteomic analysis of isolated human islets exposed to palmitate reveals time-dependent changes in insulin secretion and lipid metabolism. PLOS ONE 12 e0176391. (https://doi.org/10.1371/journal.pone.0176391)

Sargsyan E, Ortsäter H, Thorn K \& Bergsten P 2008 Diazoxide-induced beta-cell rest reduces endoplasmic reticulum stress in lipotoxic betacells. Journal of Endocrinology 199 41-50. (https://doi.org/10.1677/ JOE-08-0251)

Sargsyan E, Artemenko K, Manukyan L, Bergquist J \& Bergsten P 2016 Oleate protects beta-cells from the toxic effect of palmitate by activating pro-survival pathways of the ER stress response. Biochimica Et Biophysica Acta 1861 1151-1160. (https://doi.org/10.1016/j. bbalip.2016.06.012)

Simon-Szabó L, Kokas M, Mandl J, Kéri G \& Csala M 2014 Metformin attenuates palmitate-induced endoplasmic reticulum stress, serine phosphorylation of IRS-1 and apoptosis in rat insulinoma cells. PLoS ONE 9 e97868.

Staaf J, Ubhayasekera SJKA, Sargsyan E, Chowdhury A, Kristinsson H, Manell H, Bergquist J, Forslund A \& Bergsten P 2016 Initial hyperinsulinemia and subsequent $\beta$-cell dysfunction is associated with elevated palmitate levels. Pediatric Research 80 267-274. (https://doi.org/10.1038/pr.2016.80)

Sun Y, Ren M, Gao G, Gong B, Xin W, Guo H, Zhang X, Gao L \& Zhao J 2008 Chronic palmitate exposure inhibits AMPKalpha and decreases glucose-stimulated insulin secretion from beta-cells: modulation by fenofibrate. Acta Pharmacologica Sinica 29 443-450. (https://doi. org/10.1111/j.1745-7254.2008.00717.x)

Tucker GT, Casey C, Phillips PJ, Connor H, Ward JD \& Woods HF 1981 Metformin kinetics in healthy subjects and in patients with diabetes mellitus. British Journal of Clinical Pharmacology 12 235-246. (https:// doi.org/10.1111/j.1365-2125.1981.tb01206.x)

Viollet B, Guigas B, Sanz Garcia N, Leclerc J, Foretz M \& Andreelli F 2012 Cellular and molecular mechanisms of metformin: an overview. Clinical Science 122 253-270. (https://doi.org/10.1042/CS20110386)

Wang X, Zhou L, Li G, Luo T, Gu Y, Qian L, Fu X, Li F, Li J \& Luo M 2007 Palmitate activates AMP-activated protein kinase and regulates insulin secretion from beta cells. Biochemical and Biophysical Research Communications 352 463-468. (https://doi.org/10.1016/j. bbrc.2006.11.032)

Zhou YP \& Grill VE 1994 Long-term exposure of rat pancreatic islets to fatty acids inhibits glucose-induced insulin secretion and biosynthesis through a glucose fatty acid cycle. Journal of Clinical Investigation 93 870-876. (https://doi.org/10.1172/JCI117042)

Zhou G, Myers R, Li Y, Chen Y, Shen X, Fenyk-Melody J, Wu M, Ventre J, Doebber T, Fujii N, et al. 2001 Role of AMP-activated protein kinase in mechanism of metformin action. Journal of Clinical Investigation 108 1167-1174. (https://doi.org/10.1172/JCI13505)
Received in final form 4 June 2018

Accepted 13 June 2018 